\title{
Non-equilibrium version of the Einstein relation
}

\author{
Daniel Hurowitz, Doron Cohen \\ Department of Physics, Ben-Gurion University of the Negev, Beer-Sheva 84105, Israel
}

\begin{abstract}
The celebrated Einstein relation between the diffusion coefficient $D$ and the drift velocity $v$ is violated in non-equilibrium circumstances. We analyze how this violation emerges for the simplest example of a Brownian motion on a lattice, taking into account the interplay between the periodicity, the randomness and the asymmetry of the transition rates. Based on the non-equilibrium fluctuation theorem the $v / D$ ratio is found to be a non-linear function of the affinity. Hence it depends in a non-trivial way on the microscopics of the sample.
\end{abstract}

\section{INTRODUCTION}

The Einstein-Smoluchowski relation (ESR) [1, 2] between the diffusion coefficient $(D)$ and the mobility $(\mu)$ of a Brownian particle is a landmark in the history of statistical mechanics. It states that $D=\mu k_{B} T$, where $T$ is the temperature, and $k_{B}$ is the Boltzmann constant. Thus it reflects the microscopics of the stochastic process (via $k_{B}$ ) in a very universal way. Below we set $k_{B}=1$.

The ESR constitues the simplest example for a fluctuation-disspation relation. In a modern perspective it can be regarded as a consequence of a general nonequilibrium fluctuation theorem (NFT) [3- $[$ ] $]$ that concerns the evolving probability distribution $p(x ; t)$ that describes the stochastic motion of the particle. The ESR is in essence a relation between the second moment of the spreading $\operatorname{Var}(x)=2 D t$, and its first moment $\langle x\rangle=v t$, where $v=\mu F$ is the drift velocity, and $F$ is the field of force. Using this language it can be re-written as follows:

$$
\frac{v}{D}=f_{\sigma}(s)
$$

where $s=F / T$ is the so-called affinity in units of entropy production per unit distance, and $f_{\sigma}(s)=s$ is a universal function that does not depend on the microscopic details of the sample.

Model of interest.- We shall consider below the dynamics of a particle on an $N$ site ring, with transition rates $\vec{w}_{n}$ and $\overleftarrow{w}_{n}$ across the $n^{\text {th }}$ bond. In general the transition rates are random and asymmetric. In previous publications [7, 8] we have highlighted the relevance of Sinai spreading [9] to the analysis of the induced $v$. Optionally one may have in mind the unfolded version of our ring. The latter concerns the motion of a Brownian particle in a tilted periodic array of identically disordered unit cells.

Previous studies.- The dramatic influence of a tilt on the transport in a one-dimensional biased periodic potential has been explored experimentally for a colloidal particle on a corrugated optical vortex [10, 11], and has been exploited for optical particle fractionation and separation [12 14]. Explicit expressions for $v$ and for $D$ for the case of a tilted cosine potential were first given in [15 18] and further generalised in [19 22].

Several works have studied the effect of weak spatial disorder on the non-linear bias dependence of the transport coefficients 23 25]. Tractable expressions for $v$ and for $D$ were available for a completely disordered lattice $(N=\infty)$ [26, 27]. The prediction is that for small $s$ one obtains $v=0$. This anomaly is related to the work of Sinai [9] regarding random walk in random environment.

Strangely enough there was no attempt, as far as we know, to bridge between the implied $v / D$ dependence on $s$, and the ESR that is expected close to equilibrium. Furthermore there was no attempt to settle what looks like a contradiction with the NFT-based derivation of the ESR, which relies on the central limit theorem. It is the purpose of the present work to illuminate the departure from the ESR, and to explore the route that leads to the $N=\infty$ Sinai anomaly.

For completeness we note that extensions of the fluctuation-dissipation phenomenology far from equilibrium have been considered in 30 33], but from a different perspective. In [34] it has been pointed out that a violation of the ESR is expected in a Markovian network, however this has not been explicitly demonstrated for a model of interest, neither related to the Sinai anomaly.

\section{NFT BASED DERIVATION}

The NFT relates the probability of a stochastic trajectory $\boldsymbol{r}(t)$ to the probability of the time reversed process:

$$
\frac{P[\boldsymbol{r}(-t)]}{P[\boldsymbol{r}(t)]}=\exp [-\mathcal{S}[\boldsymbol{r}]]
$$

where $\mathcal{S}[\boldsymbol{r}]$ is the entropy production that is associated with the trajectory. The implicit assumption here is that $\mathcal{S}[\boldsymbol{r}]$ is well defined. This is a very strong assumption because in an actual experiment $\mathcal{S}$ might depend on additional "hidden" microscopic coordinates that cannot be resolved by the measuring device. This can be summarized by saying that coarse-graining might make the "bare" NFT inapplicable: possibly an effective version of $\mathcal{S}[\boldsymbol{r}]$ should be defined [35 37]. In our model $\mathcal{S}[\boldsymbol{r}]$ is a well defined functional, still we shall see later that in some sense $s$ is renormalized due to coarse-graining.

We proceed with a critical overview of the the derivation of the traditional ESR based on Eq.(2). The entropy production during one trip around the ring is

$$
\mathcal{S}_{\circlearrowleft}=\sum_{n \in \text { ring }} \ln \left[\frac{\vec{w}_{n}}{\bar{w}_{n}}\right]
$$


The terms in this sum can be regarded as a stochastic field. Summing over the terms along the closed loop we get the stochastic motive force (SMF). In the context of molecular motors the SMF is known as the affinity. The entropy production for a general trajectory that has a winding number $q$ is $\mathcal{S}[r]=q \mathcal{S}_{\circlearrowleft}$. We disregard here small fractional-loop error that can be neglected in the infinite time limit. We define formally the distance as $x=q N$, and the entropy per unit distance as $s=\mathcal{S}_{\circlearrowleft} / N$. If follows from Eq. (2) that the evolving probability distribution satisfies

$$
\frac{p(-x ; t)}{p(x ; t)}=\mathrm{e}^{-s x}
$$

In the long time limit, by virtue of the central limit theorem (CLT), one can introduce a Gaussian approximation $p(x) \approx \bar{p}(x)$, where

$$
\bar{p}(x ; t)=\frac{1}{\sqrt{4 \pi D t}} \exp \left[-\frac{(x-v t)^{2}}{4 D t}\right]
$$

Substitution in Eq.(4) leads to the standard ESR, namely $v / D=s$. Below we are going to argue that the last step should be handled with much more care. The coarsegrained distribution $\bar{p}(x)$ that appears in the CLT, is in fact a convoluted ("smoothed") version of the bare $p(x)$. If follows that $\bar{p}(x)$ obeys a "dressed" version of Eq. (44), with effective affinity $\bar{s}=f_{\sigma}(s)$, where

$$
f_{\sigma}(s)=\frac{2}{a_{s}} \tanh \left(\frac{a_{s} s}{2}\right)
$$

The length scale $a_{s}$ is related to the microscopic details of the model. We first clarify this statement for the simplest case of a non-disordered ring, for which $a_{s}=1$ is the lattice constant, and later discuss the general case.

At this point one should realize that Eq.(1) with Eq.(6) can be regarded as a generalized ESR. The $v / D$ ratio is no longer a universal function because there is an affinity dependent length-scale $a_{s}$, that reflects the microscopic structure. The standard ESR is recovered if $a_{s} s \ll 1$. In the other extreme we get the simple relation

$$
D=\frac{1}{2} v_{s} a_{s}
$$

where the subscript $s$ has been added in order to emphasize the crucial dependence on the affinity. We would like to illuminate how $a_{s}$ depends on the periodicity $N$, on the strength of the disorder $\sigma$, and on the affinity $s$.

\section{EFFECTIVE AFFINITY}

Consider the simplest discrete model with asymmetry. All the bonds are identical; the transition rates from left to right are $\vec{w}$, and the transition rates from right to left are $\overleftarrow{w}$. Hence it follows from Eq.(3) that $\mathcal{S}_{\circlearrowleft}=\ln (\vec{w} / \overleftarrow{w})$. It is not difficult to find the exact expression for the the evolving probability distribution $p(x ; t)$. The dynamics that is generated by a rate equation can be simulated as a random walk process with infinitesimal time steps $\tau$. The traversed distance is $x=X_{1}+\ldots+X_{\mathcal{N}}$. The transition probabilities per step are

$$
\begin{aligned}
P(X=+1) & =p \equiv \vec{w} \tau \\
P(X=-1) & =q \equiv \overleftarrow{w} \tau \\
P(X=0) & =1-p-q
\end{aligned}
$$

The probability distribution can be obtained from the moment generating function of the process

$$
Z(k)=\left\langle\mathrm{e}^{-i k x}\right\rangle=\left[p \mathrm{e}^{-i k}+q \mathrm{e}^{+i k}+(1-p-q)\right]^{\mathcal{N}}
$$

In the continuous time limit $p, q \ll 1$, hence one can expand

$$
\ln Z(k)=\mathcal{N}\left[p \mathrm{e}^{-i k}+q \mathrm{e}^{+i k}-(p+q)\right]+\mathcal{O}\left(\mathcal{N} \tau^{2}\right)
$$

Accordingly, one obtains

$$
p(x ; t)=\int_{-\infty}^{\infty} d k \mathrm{e}^{i k x+\left(\vec{w} \mathrm{e}^{-i k}+\overleftarrow{w} \mathrm{e}^{i k}-(\overleftarrow{w}+\vec{w})\right) t}
$$

This distribution obviously satisfies the NFT Eq. (4), which can be easily verified by inverting the sign of the dummy integration variable in $p(-x ; t)$ and then shifting it by a constant $k \rightarrow k+\ln (\overleftarrow{w} / \vec{w})$. Expanding the expression in the exponent in powers of $k$ one obtains

$$
p(x ; t)=\int_{-\infty}^{\infty} d k \mathrm{e}^{i k(x-(\vec{w}-\overleftarrow{w}) t)-\frac{k^{2}}{2}(\vec{w}+\overleftarrow{w}) t+\mathcal{O}\left(k^{3} t\right)}
$$

The average $v t$ and the variance $2 D t$ are implied by the coefficients of the $k$ and $k^{2}$ terms in the exponent, namely

$$
\begin{aligned}
v & =(\vec{w}-\overleftarrow{w}) \\
D & =\frac{1}{2}(\vec{w}+\overleftarrow{w})
\end{aligned}
$$

The $v / D$ ratio is given by Eq.(6) with $a_{s}=1$. This exact result clearly contradicts the traditional ESR.

We now would like to see what happens to the NFT and the ESR if the CLT is applied. Recall that the CLT procedure is to introduce the re-scaled variable $(x-v t) /(2 D t)$ and to claim that in the $t \rightarrow \infty$ limit the higher order cumulants $\mathcal{O}\left(k^{3} t\right)$ can be neglected. We use the notation $\bar{p}(x ; t)$ for the normal distribution that is obtained via CLT. It is easy to see that it satisfies Eq. (4), but with an effective value of $s$ that is given by Eq.(6). Consequently the associated relation $v / D=\bar{s}$ is consistent with the exact analysis, while the bare relation $v / D=s$ is violated.

We conclude that the normal approximation for $p(x ; t)$, which is implied by CLT, obeys the NFT provided $s$ is replaced by a renormalized value $\bar{s}$. The reason for that is as follows: The CLT procedure is the same as cutting off the high $k$ modes, which is the same as smoothing the function $p(x, t)$. Due to the smoothing the effective value of $s$ becomes smaller, and consequently the bare ESR is violated. 


\section{DRIFT AND DIFFUSION}

We now turn to describe a general procedure for exact calculation of $v$ and $D$. Any rate equation can be written schematically as $d \boldsymbol{p} / d t=W \boldsymbol{p}$, where $\boldsymbol{p}=\left\{p_{n}\right\}$ is a column vector that contains the occupation probabilities, and $W$ is a matrix that contains the transition rates. Note that this matrix is non-symmetric, hence one should distinguish between right and left eigenvectors. If the lattice is periodic, with a unit cell that consists of $N$ sites, the eigenevectors satisfy the Bloch theorem. The reduced equation for the eigenmodes becomes $\boldsymbol{W}(\varphi) \psi=-\lambda \psi$, where $\boldsymbol{W}(\varphi)$ is an $N \times N$ matrix, and the presence of the phase $\varphi$ implies that $\psi_{n+N}=\mathrm{e}^{i \varphi} \psi_{n}$, where $n$ is the site index $\bmod (N)$. The Bloch quasi-momentum is formally defined via the relation $\varphi \equiv k N$. The outcome of the diagonalization process are the Bloch state $|k, \nu\rangle$, where $\nu$ is the band index, and the corresponding eigenvalues are $-\lambda_{\nu}(k)$. The bottom line is that the time dependent solution of the rate equation can be written as

$$
p_{n}(t) \approx \frac{1}{L} \sum_{k, \nu} C_{k, \nu} \mathrm{e}^{-\lambda_{\nu}(k) t} \mathrm{e}^{i k n}
$$

where $C_{k, \nu}$ are constants that depend on the initial preparation. For technical details see appendix A. The long time spreading is dominated by the lowest band $\nu=0$. It is not difficult to show (see appendix) that the drift velocity and the diffusion coefficient are determined by the derivatives of $\lambda_{0}(k)$. Namely,

$$
\begin{aligned}
v & =\left.i \frac{\partial \lambda_{0}(k)}{\partial k}\right|_{k=0} \\
D & =\left.\frac{1}{2} \frac{\partial^{2} \lambda_{0}(k)}{\partial k^{2}}\right|_{k=0}
\end{aligned}
$$

The $N=2$ system. - As an explicit example for the outcome of this procedure we consider a periodic lattice that has unit cell with $N=2$ sites. The transition rates $(\vec{A}, \overleftarrow{A}, \vec{B}, \overleftarrow{B})$ are characterized by $\ln (\vec{A} / \overleftarrow{A})=s+\sigma$ and $\ln (\vec{B} / \overleftarrow{B})=s-\sigma$, such that $\sigma$ is the "disorder" (in a later example $\sigma$ will stand for the width of a box distribution). After some straightforward algebra (see Appendix B) one obtains

$$
v=\frac{\vec{A} \vec{B}-\overleftarrow{A} \overleftarrow{B}}{\vec{A}+\overleftarrow{A}+\vec{B}+\overleftarrow{B}}
$$

and

$$
D=\frac{1}{2}\left[\frac{\vec{A} \vec{B}+\overleftarrow{A} \overleftarrow{B}-2 v^{2}}{\vec{A}+\overleftarrow{A}+\vec{B}+\overleftarrow{B}}\right]
$$

The $v / D$ ratio is given by Eq.(11) with

$$
f_{\sigma}(s)=\frac{2}{1+\tanh ^{2}\left(\frac{\sigma}{2}\right) \tanh ^{2}\left(\frac{s}{2}\right)} \tanh \left(\frac{s}{2}\right)
$$

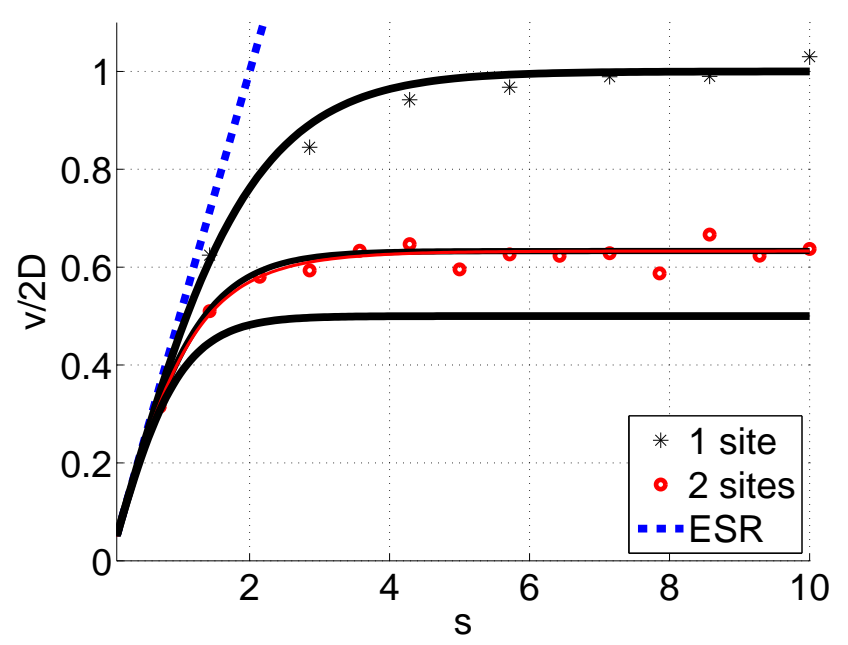

FIG. 1: The ratio $v /(2 D)$ for a Brownian motion in a onedimensional regular lattice. The number of sites per unit cell is $N=1$ (black stars) and $N=2$ (red circles). The numerical results (symbols) are based on simulations with ensembles of $10^{3}$ trajectories, while the lines are exact analytical expressions. The dashed line is the ESR. The upper and lower thick solid lines are Eq.(6) with $a=1$ and $a=2$ respectively. In the $N=2$ case $\overleftarrow{A} \vec{A}=\overleftarrow{B} \vec{B}=1$ and $\sigma=2$. The intermediate thick solid line is Eq. (6) with $a=a_{\infty}=1.58$. It barely can be resolved from the exact result (thin red line).

This result is compared to a numerical simulation of a random walk in Fig.1 which was obtained by standard simulation methods (Gillespie's algorithm). As $s$ is increased the $v / D$ ratio approaches a limiting value which we define as $2 / a_{\infty}$. In Fig.1 1 we have added a curve of the function Eq. (6) with $a_{s}=a_{\infty}$. We observe that for practical purpose $a_{\infty}$ can be regarded as an effective lattice constant. As the "disorder" $\sigma$ increases $\tanh ^{2}(\sigma / 2)$ grows from 0 to 1 , and consequently $a_{\infty}$ grows from the value $a=1$ to the value $a=2$. In spite of the simplicity of this example we shall see that it provides partial insight with regard to the general $N$ case.

General $N$ system.- Let us explore how $f_{\sigma}(s)$ looks like when $N$ becomes larger. Fig 2 provides a few examples that were calculated analytically using Eq. (18) and Eq.(19) for $N=20$. The rates were chosen as $\vec{w}_{n}=\mathrm{e}^{\mathcal{S}_{n} / 2}$ and $\overleftarrow{w}_{n}=\mathrm{e}^{-\mathcal{S}_{n} / 2}$, where $\mathcal{S}_{n}$ are box distributed within $[s-\sigma, s+\sigma]$. This implies that the rates have log-box distribution as in "glassy" systems.

Looking at the numerical results (Fig.21), and taking into account the simple periodic lattice as a reference case, we observe that $f_{\sigma}(s)$ has some typical properties that we would like to analyze in later sections. Namely: (1) For small values of $s$ we have $f_{\sigma}(s)=s$ in consistency with the ESR. (2) For no disorder $(\sigma=0)$ we already have established that $f_{\sigma}(s)$ obeys Eq. (6) with $a_{s}=1$, reflecting the microscopic discreteness of the lattice. (3) For finite disorder we see that for moderate values of $s$ the function $f_{\sigma}(s)$ can be approximated by Eq.(6) with $a_{s}=N$, reflecting the length of the unit cell. 


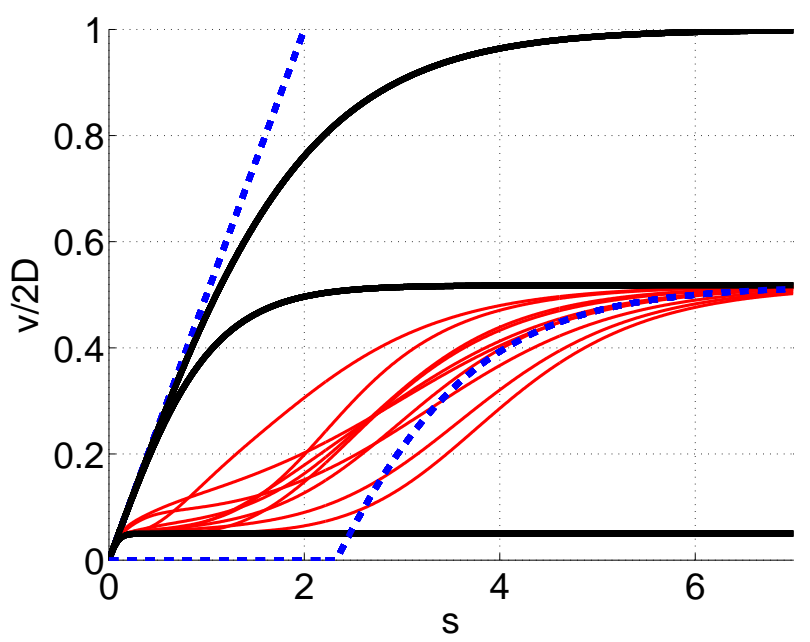

FIG. 2: The same as Fig 1 but with $N=20$ sites per unit cell. The upper and lower thick solid lines are for zero disorder $(\sigma=0)$ and for infinite disorder $(\sigma \rightarrow \infty)$, as implied by Eq. (6) with $a=1$ and $a=20$ respectively. The thin solid curves are based on exact analytical calculation for various realizations of disorder that is characterized by $\sigma=3.5$. The intermediate thick solid line is Eq. (6) with $a=a_{\infty}=1.9316$, estimated using Eq. (29). The linear dashed line is the ESR while the second dashed line that exhibits a "Sinai step" is the $N=\infty$ prediction of [26] (see text).

(4) For very large values of $s$ the function $f_{\sigma}(s)$ saturates, reflecting an effective lattice constant $a_{\infty}$ that we discuss in Section V. (5) As $N$ becomes larger our results approach those of [26], as discussed in Section VII.

\section{THE POISSON LIMIT}

Going to the extreme of very large $s$ it is possible to get simple analytical expressions for $v$ and $D$. We first consider a simple periodic lattice. From Eq.(15)-Eq.(16) we get $v=\vec{w} a$ and $D=(1 / 2) \vec{w} a^{2}$ where $a=1$ is the lattice constant. Accordingly $v /(2 D)=1$ in consistency with Eq.(17). Let us illuminate the statistical meaning of this result. Recall that the dynamics is generated by a rate equation that can be simulated as a random walk process with infinitesimal time steps $\tau$. The traversed distance is $x=X_{1}+\ldots+X_{N}$. For $s \rightarrow \infty$ we get a Poisson process

$$
\begin{aligned}
P(X=1) & =p \\
P(X=0) & =1-p \\
P(X=-1) & =0
\end{aligned}
$$

with $p=\vec{w} \tau$. Taking the continuous time limit one deduces that in the Poisson limit the ratio between the first and the second moment is unity, hence $v /(2 D)$ is determined.

We now turn to the disordered case, i.e. the rates are not the same. In the Poisson limit we still can get a simple analytical expression for $a_{\infty}$, which determines

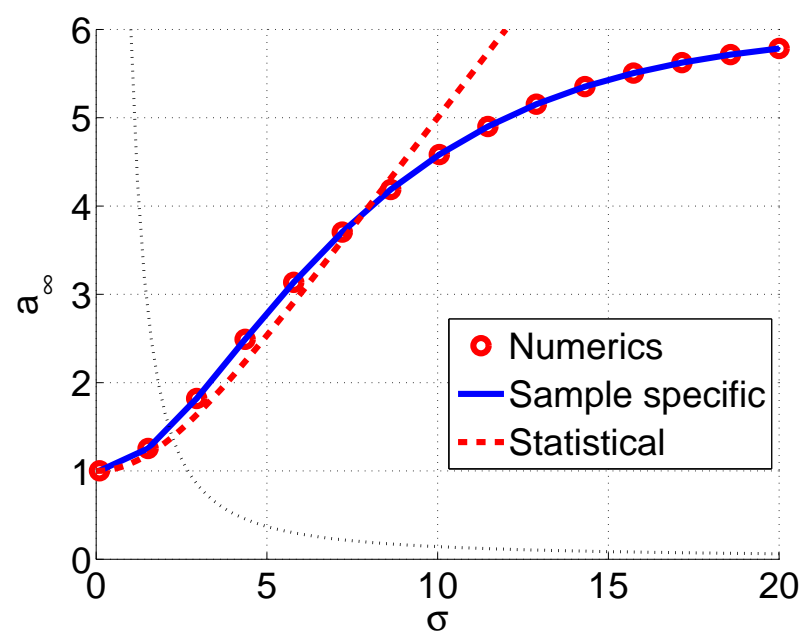

FIG. 3: The effective length scale $a_{\infty}=(2 D / v)_{s \rightarrow \infty}$. The number of sites per unit cell is $N=6$. The symbols are the outcome of an exact analytical calculation. The exact sample specific expression Eq. (29) (solid line) works perfectly, independent of $N$. The statistical approximation Eq. (30) (dashed line) becomes indistinguishable for large values of $N$ (not shown). The dotted line is $a^{(\sigma)}$ of Eq. (39).

the asymptotic value of $v /(2 D)$. This is done using the same procedure as in section III. The rates are $\vec{w}_{n}=w_{n}$ and $\overleftarrow{w}_{n}=0$ for $n=1 \ldots N$. The characteristic equation for the eigenvalues of $W(\varphi)$ is

$$
\operatorname{det}(\lambda+\boldsymbol{W}(\varphi))=\prod_{n=1}^{N}\left(\lambda-w_{n}\right)+\mathrm{e}^{-i \varphi} \prod_{n=1}^{N} w_{n}=0
$$

This can be re-written as

$$
\prod_{n=1}^{N}\left(1-\frac{\lambda}{w_{n}}\right)=\mathrm{e}^{-i \varphi}
$$

Expanding to second order we get

$$
\sum_{n=1}^{N} \frac{\lambda}{w_{n}}-\sum_{i \neq j} \frac{\lambda^{2}}{w_{n} w_{m}}=1-\mathrm{e}^{-i \varphi}
$$

with the solution

$$
\begin{aligned}
\lambda & =-i\left[\left(\sum_{n=1}^{N} \frac{1}{w_{n}}\right)^{-1}\right] \varphi \\
& +\frac{1}{2}\left[\left(\sum_{n=1}^{N} \frac{1}{w_{n}}\right)^{-3}\left(\sum_{n=1}^{N} \frac{1}{w_{n}^{2}}\right)\right] \varphi^{2}+\mathcal{O}\left(\varphi^{3}\right)
\end{aligned}
$$

Using Eq.(18) and Eq.(19) we deduce that

$$
a_{\infty}=\left(\frac{2 D}{v}\right)_{s \rightarrow \infty}=\left[\frac{\left\langle(1 / \vec{w})^{2}\right\rangle}{\langle(1 / \vec{w})\rangle^{2}}\right]
$$


where the sample average is $\langle R\rangle \equiv(1 / N) \sum_{n} R_{n}$. For large $N$ the sample average can replaced by an ensemble average. Note that the expression in the square brackets constitutes a measure for the "glassiness" of the network: it becomes much larger than unity due to the presence of weak links. For the log-box distributed transition rates of Fig 2

$$
a_{\infty}=\frac{\sigma}{2} \operatorname{coth}\left(\frac{\sigma}{2}\right)
$$

In Fig 3 we test this estimate for $N=6$. We observe (not shown) that the statistical result Eq. (30) becomes indistinguishable from the exact sample average Eq. (29) for large values of $N$.

\section{DIGRESSION - SINAI SPREADING}

In order to understand the dependence of $v$ and $D$ on $N$ and $s$, it is useful to recall known results that have been obtained for the time-dependent spreading in an $N=\infty$ lattice. Recall that the drift is induced by the stochastic field, whose affinity is defined in Eq.(3) ). The comulant generating function of the stochastic field can be written as $g(\mu)=\left(s-s_{\mu}\right) \mu$, where $s_{\mu}$ is defined via the following expression:

$$
\left\langle\left(\frac{\overleftarrow{w}}{\bar{w}}\right)^{\mu}\right\rangle \equiv \mathrm{e}^{-\left(s-s_{\mu}\right) \mu}
$$

If the stochastic field has normal distribution with standard deviation $\sigma$, then $s_{\mu}=(1 / 2) \sigma^{2} \mu$. For our box distribution

$$
s_{\mu}=\frac{1}{\mu} \ln \left(\frac{\sinh (\mu \sigma)}{\mu \sigma}\right)
$$

which is monotonically ascending from zero to $\sigma$. The positive monotonic function $s_{\mu}$ can be inverted, hence we can define a scaled affinity $\mu(s)$. Note that Eq. (31) implies that $\mu(s)$ is the value of $\mu$ for which the left-handside equals unity.

The time dependent spreading in the zero bias case $(s=0)$ has been worked out by Sinai $([\underline{9}])$, leading to the anomalous time dependence

$$
x \sim[\log (t)]^{2}
$$

For finite $s$ one obtains 28? ]

$$
x \sim t^{\mu}
$$

where the exponent $\mu=\mu(s)$ is the "scaled affinity" that has been defined above.

From the time dependent spreading we can deduce the $N$ dependence of the stationary drift velocity. The deduction goes as follows: The time required to drift a distance $x \sim N$ is $t \sim N^{1 / \mu}$, hence

$$
v \sim \frac{x}{t} \sim\left(\frac{1}{N}\right)^{\frac{1}{\mu}-1}
$$

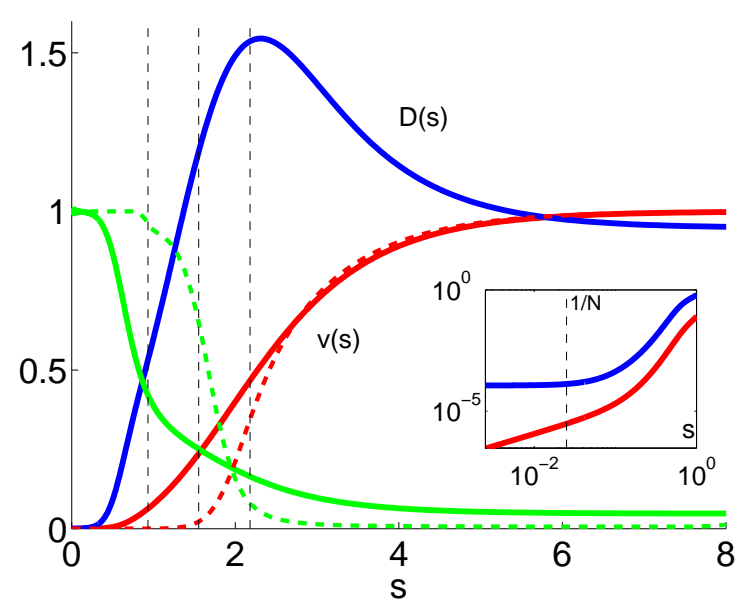

FIG. 4: The drift velocity $v_{s}=v(s)$ [labeled red lines], the monotonic decreasing $a_{s} / N$, and the diffusion coefficient $D=D(s)$ [labeled blue lines] as a function of $s$. The time units are chosen such that $v_{\infty}=\langle 1 / \vec{w}\rangle^{-1}=1$. The horizontal lines from left to right correspond to $s=1 / N$ (inset), and $s_{1 / 2}$, and $s_{1}$, and $s_{2}$. They divide the $s$ dependence into 5 distinct regimes (see text). Solid lines are for $N=20$ and $\sigma=3.5$. The dashed lines are for $N=300$. For the latter the step in $v\left(\right.$ at $\left.s=s_{1}\right)$ and the drop in $a$ (at $s=s_{2}$ ) become more pronounced.

This result has meaning only within the Sinai regime $s<s_{1}$. One observed that the dependence of $v$ on $N$ is either sub-Ohmic or super-Ohmic depending whether $s$ is smaller or larger than $s_{1 / 2}$. Note that for $s \sim 0$ the same argument leads to the well known Sinai suppression factor $\sim \exp (-\sqrt{N})$ that reflects the build-up of an activation barrier.

\section{THE SINAI ANOMALY}

For a non-periodic disordered lattice $(N=\infty)$ it has been found [26] that outside of the Sinai regime $\left(s>s_{1}\right)$ the the drift velocity is

$$
v_{s}=\frac{1-\langle(\overleftarrow{w} / \vec{w})\rangle}{\langle(1 / \vec{w})\rangle}=\left[1-\mathrm{e}^{-\left(s-s_{1}\right)}\right] v_{\infty}
$$

The exact expression for the diffusion coefficient is also known but is quite lengthy [26]. In practice we find that we can deduce $D$ from Eq.(17) if we make the identification

$$
a_{s} \approx \frac{a_{\infty}}{1-\left\langle(\overleftarrow{w} / \vec{w})^{2}\right\rangle}=\frac{a_{\infty}}{1-\mathrm{e}^{-2\left(s-s_{2}\right)}}
$$

This expression holds in the normal diffusion regime $s>s_{2}$, where $D$ is finite. We emphasize again that this expression is implied by inspection of a very complicated formal expression [26] whose physics we would like to illuminate below. In a practical perspective our approach 


\begin{tabular}{|l||c|c|c|c|c|}
\hline$s$ regime & {$[0,1 / N]$} & {$\left[1 / N, s_{1 / 2}\right]$} & {$\left[s_{1 / 2}, s_{1}\right]$} & {$\left[s_{1}, s_{2}\right]$} & {$\left[s_{2}, \infty\right]$} \\
\hline$a_{s}$ & irrelevant & \multicolumn{2}{|c|}{$a_{s} \sim N$} & \multicolumn{2}{|c|}{$a_{s} \approx\left[1-\mathrm{e}^{-2\left(s-s_{2}\right)}\right]^{-1} a_{\infty}$} \\
\hline$v_{s}$ & $v=2 D s$ & $\sim\left(\frac{1}{N}\right)^{\frac{1}{\mu}-1}$ & \multicolumn{2}{|r|}{$v_{s} \approx\left[1-\mathrm{e}^{-\left(s-s_{1}\right)}\right] v_{\infty}$} \\
\hline$D$ & $\sim \exp (-\sqrt{N})$ & $\sim(1 / N)^{\frac{1}{\mu}-2}$ & $\sim(N)^{2-\frac{1}{\mu}}$ & $\sim N$ & $D=\frac{1}{2} a_{s} v_{s}$ \\
\hline
\end{tabular}

TABLE I: Transport characteristics in the various $s$ regimes. In the $s<s_{1}$ regime the transport is suppressed due to Sinai activation-barrier that scales with $N$. In the $s>s_{1}$ regime the bias stretches away this barrier, and the drift approaches its resistor-network limited value $v_{\infty}$. Normal diffusion is recovered for $s>s_{2}$.

opens the way for an easy extension to finite $N$, which we present in the next section.

The result of the $v / D$ calculation using Eq. (77) with Eq. (37) is displayed in Fig.2. Due to the Sinai anomaly we have $v=0$ within a finite range $s<s_{1}$, hence the ESR is completely violated. For large but finite $N$ we observe in Fig.2 the remnants of the Sinai anomaly, which we call "Sinai step". The question arises what does it mean "large $N$ ". For this purpose let us use a hand-waving argument in order to illuminate the reason for having a vanishingly small drift velocity. In a quasi-equilibrium situation we have $\vec{w}_{n} p_{n}=\overleftarrow{w}_{n} p_{n+1}$. It follows that $p_{n} \sim$ $\alpha^{n}$ where

$$
\alpha \equiv\left\langle\frac{\overleftarrow{w}}{\vec{w}}\right\rangle^{-1}=\mathrm{e}^{s-s_{1}}
$$

Transport into the sample is possible provided $\alpha>1$, else, if $[\log (1 / \alpha)] N \gg 1$, the probability for penetration is blocked. Thus a small $s$ regime with vanishingly small drift velocity is feasible if $N \gg a^{(\sigma)}$, where

$$
a^{(\sigma)}=\frac{1}{s_{1}}=\left[\ln \left(\frac{\sinh \sigma}{\sigma}\right)\right]^{-1}
$$

The dependence of $a^{(\sigma)}$ on $\sigma$ is illustrated in Fig 3 In the numerics we assume large $\sigma$ values, so there are remnants of the Sinai anomaly. For weak disorder $(\sigma \ll 1)$ only a very large sample will exhibit the "Sinai step" that we see in Fig,2,

\section{THE $s$ DEPENDENCE OF $D$}

The generalized ESR Eq. (11) with the affinitydependent length scale $a(s)$ can be used in order to deduce the dependence of $D$ on $s$ for a finite $N$ system with disorder. For $s<1 / N$ we have the conventional linear dependence that is predicted by the traditional ESR. For $s>1 / N$ the diffusion coefficient $D$ is determined by the product $v_{s} a_{s}$, see Eq. (7).

Recall that the $N$ dependence of $v_{s}$ goes from subOhmic to super-Ohmic at $s=s_{1 / 2}$, and becomes $N$ independent for $s>s_{1}$. Recall also that $a_{s}$ scales like $N$ for $s<s_{2}$, and becomes $N$ independent for $s>s_{2}$. Accordingly as $s$ increases we have the following regimes: (1) Linear hopping regime for $s<1 / N$. (2) Hopping regime with small $D$ for $s \in\left[1 / N, s_{1 / 2}\right]$. (3) Hopping regime with large $D$ for $s \in\left[s_{1 / 2}, s_{1}\right]$. (4) Sliding regime with large $D$ for $s \in\left[s_{1}, s_{2}\right]$. (5) Sliding regime with normal diffusion for $s>s_{2}$. We use the keywords "Hopping" and "Sliding" in order to indicate whether $v_{s}$ vanishes or not in the $N \rightarrow \infty$ limit. We use the keywords "small" and "large" with regard to $D$ in order to indicate whether it vanishes or diverges in the $N \rightarrow \infty$ limit, while "normal" means finite result in this limit. The generic $s$ dependence is illustrated in Fig 4 and summarized in Table I.

\section{DISCUSSION}

In general non-equilibrium circumstances the bare ESR is not valid. For the model system of interest we write its generalized version as Eq. (11) with Eq. (6), where $a_{s}$ is an $s$ dependent effective scale that depends on microscopics of the sample. It is only in the quasiequilibrium limit $s \rightarrow 0$ that the traditional ESR becomes valid. As the affinity $s$ is increased, the length-scale $a_{s}$ drops from the maximal value $a_{0}=N$ (the periodicity) to the disorder limited value value $a_{\infty}$. The latter depends monotonically on the strength $\sigma$ of the disorder.

The first impression is that the generalized ESR Eq.(1) with Eq.(6) is very wrong. Naively the ESR should apply also in non-equilibrium circumstances because it can be derived from the NFT assuming CLT. We have explained that the resolution of this puzzle is related to the implicit coarse-graining procedure. Consequently the effective affinity is $\bar{s}=f_{\sigma}(s)$. One wonders what is the "small parameter" on which the ESR is based. Considering Brownian motion on an $a=1$ periodic lattice the answer is that the affinity should be small $(s a \ll 1)$. For a disordered lattice with period $N$ the effective lattice constant $a_{s}$ becomes larger, and hence the condition $s a_{s} \ll 1$ becomes more demanding.

The implication of coarse graining is relevant experimentally if the resolution of the measurement apparatus is limited 35]. A recent example presented itself in an experimental test of the NFT for electron transport through a quantum dot 38 40]. The explanation of the apparent violation of the NFT has been based on the elimination of secondary loops in the circuit [36, 37], hence what counts is not the bare affinity but the effective affinity. In the present study we have assumed that topological ambiguities are absent. There are no secondary loops, just simply connected circuit. Still 
we see that the NFT cannot be applied using the bare affinity. Replacing it by an effective affinity one obtains the generalized ESR.
Acknowledgments.- This research was supported by the Israel Science Foundation (grant No.29/11). We thank Saar Rahav (Technion) for motivating discussions, and Pierre Gaspard (ULB) for a useful advice.
[1] A. Einstein, Annalen der Physik 322, 549 (1905).

[2] M. Smoluchowski, Annalen der Physik 326, 756 (1906).

[3] J. L. Lebowitz, H. Spohn, J. Stat. Phys. 95, 333 (1999).

[4] P. Gaspard, J. Chem. Phys., 120, 8898 (2004).

[5] U. Seifert, Phys. Rev. Lett. 95, 040602 (2005).

[6] D. Andrieux, P. Gaspard, J. Stat. Phys., 127, 107 (2007).

[7] D. Hurowitz, S. Rahav, D. Cohen, Europhys. Lett. 98, 20002 (2012).

[8] D. Hurowitz, S. Rahav, D. Cohen, Phys. Rev. E. 88, 062141 (2013).

[9] Ya.G. Sinai, Theory Probab. Appl. 27, 256 (1982).

[10] S.H. Lee, D.G. Grier, Phys. Rev. Lett. 96, 190601 (2006).

[11] M. Evstigneev, O. Zvyagolskaya, S. Bleil, R. Eichhorn, C. Bechinger, P. Reimann, Phys. Rev. E. 77(4), 041107 (2008).

[12] M.P. MacDonald, G.C. Spalding, K. Dholakia, Nature, 426(6965), 421-424 (2003).

[13] K. Ladavac, K. Kasza, D.G. Grier, Phys. Rev. E 70(1), 010901 (2004).

[14] G. Milne, D. Rhodes, M. MacDonald, K. Dholakia, Optics letters, 32(9), 1144-1146 (2007).

[15] H. Risken, The Fokker Planck Equation (Springer Berlin 1984).

[16] G. Costantini, F. Marchesoni, Europhys. Lett. 48, 491 (1999)

[17] M. Borromeo, F. Marchesoni, CHAOS 15, 026110 (2005)

[18] C. Cattuto, F. Marchesoni Phys. Rev. Lett. 79, 5070 (1997)

[19] P. Reimann, C. Van den Broeck, H. Linke, P. Hanggi, J. M. Rubi, A. Perez-Madrid, Phys. Rev. Lett. 87(1), 010602 (2001).

[20] P. Reimann, C. Van den Broeck, H. Linke, P. Hanggi, J. M. Rubi, A. Perez-Madrid, Phys. Rev. E. 65, 031104 (2002).

[21] B. Lindner, M. Kostur, L. Schimansky-Geier, Fluctuation and Noise Letters, 1, R25 (2001).

[22] V. Blickle, T. Speck, U. Seifert, C. Bechinger Phys. Rev. E. 75, 060101 (2007).

[23] K. Lindenberg, J.M. Sancho, M. Khoury, A.M. Lacasta, Fluctuation and Noise Letters 11, No.1, 1240004 (2012).

[24] P. Reimann, R. Eichhorn, Phys. Rev. Lett. 101(18), 180601 (2008).

[25] M. Khoury, A.M. Lacasta, J.M. Sancho, K. Lindenberg, Phys. Rev. Lett. 106, 090602 (2011).

[26] B. Derrida, J. Stat. Phys. 31, 3, (1983).

[27] C. Aslangul, N. Pottier, D. Saint-James, J. Phys. France 50, 899-921 (1989).

[28] J.P. Bouchaud, A. Comtet, A. Georges, P. Le Doussal, Annals of Physics, 201, 285-341 (1990).

[29] J.P. Bouchaud, A. Georges, Phys. Rep. 195, 127-293 (1990)

[30] V. Blickle, T. Speck, C. Lutz, U. Seifert, and C. Bechinger, Phys. Rev. Lett. 98, 210601 (2007).

[31] U. Seifert, T. Speck, Europhys. Lett. 89, 10007 (2010).

[32] T. Speck, U. Seifert, Europhys. Lett. 74, 391 (2006).
[33] D. Hurowitz, D. Cohen, Europhys. Lett. 93, 60002 (2011).

[34] U. Seifert, Phys. Rev. Lett. 104, 138101 (2010).

[35] S. Rahav, C. Jarzynski, J. Stat. Mech. P09012 (2007).

[36] G.B. Cuetara, M. Esposito, P. Gaspard, Phys. Rev. B 84, 165114 (2011).

[37] G. B. Cuetara, M. Esposito, G. Schaller, P. Gaspard, Phys. Rev. B 88, 115134 (2013).

[38] S. Nakamura, Y. Yamauchi, M. Hashisaka, K. Chida, K. Kobayashi, T. Ono, R. Leturcq, K. Ensslin, K. Saito, Y. Utsumi, A.C. Gossard, Phys. Rev. Lett. 104, 080602 (2010)

[39] S. Nakamura, Y. Yamauchi, M. Hashisaka, K. Chida, K. Kobayashi, T. Ono, R. Leturcq, K. Ensslin, K. Saito, Y. Utsumi, A.C. Gossard, Phys. Rev. B 83, 155431 (2011)

[40] B. Kung, C. Rossler, M. Beck, M. Marthaler, D. S. Golubev, Y. Utsumi, T. Ihn, K. Ensslin, Phys. Rev. X 2, 011001 (2012) 


\section{Appendix A: Procedure for calculating $v$ and $D$}

We present here the general procedure for calculating $v$ and $D$ of a diffusive particle on a lattice that has an $N$ site unit cell. For presentation purpose let us consider for example a lattice with a 2 site unit cell. The rate equation for (say) sites $n=3,4$ takes the form

$$
\begin{aligned}
& \dot{p}_{3}=\vec{A} p_{2}-(\overleftarrow{A}+\vec{B}) p_{3}+\overleftarrow{B} p_{4} \\
& \dot{p}_{4}=\vec{B} p_{3}-(\overleftarrow{B}+\vec{A}) p_{4}+\overleftarrow{A} p_{5}
\end{aligned}
$$

where $p_{n}$ are the occupation probabilities of the infinite lattice. Applying Bloch theorem the right eigenvectors are determined by two amplitudes $\psi_{1}$ and $\psi_{2}$, and the recursion $\psi_{n+2}=\mathrm{e}^{i \varphi} \psi_{n}$, where the Bloch phase $\varphi \equiv k N$ is used to define the quasi-momentum $k$. The reduced equation for the Bloch amplitudes is

$$
\left(\begin{array}{cc}
-(\overleftarrow{A}+\vec{B}) & \vec{A} \mathrm{e}^{-i \varphi}+\overleftarrow{B} \\
\vec{B}+\overleftarrow{A} \mathrm{e}^{i \varphi} & -(\overleftarrow{B}+\vec{A})
\end{array}\right)\left(\begin{array}{c}
\psi_{1} \\
\psi_{2}
\end{array}\right)=-\lambda\left(\begin{array}{c}
\psi_{1} \\
\psi_{2}
\end{array}\right)
$$

The minus sign in front of the eigenvalues is a matter of convention. Note that for $\varphi=0$ one obtains the lowest eigenvalue $\lambda_{0}=1$ which is associated with the NESS. Schematically we write the reduced equation as $\boldsymbol{W} \psi=-\lambda \psi$. The generalization for $N$ site unit cell is straightforward. Using Dirac notations the reduced Bloch equation is

$$
\boldsymbol{W}(\varphi)|\varphi, \nu\rangle=-\lambda_{\nu}(\varphi)|\varphi, \nu\rangle
$$

Since $\boldsymbol{W}$ is not a symmetric matrix, one should distinguish between left and right eigenvectors. The left eigenvectors are defined via the equation

$$
\langle\varphi, \tilde{\nu}| \boldsymbol{W}(\varphi)=-\lambda_{\nu}(\varphi)\langle\varphi, \tilde{\nu}|
$$

Optionally the latter can be regarded as the right eigenvectors of $\mathbf{W}^{\dagger}$

$$
\boldsymbol{W}^{\dagger}(\varphi)|\varphi, \tilde{\nu}\rangle=-\lambda_{\nu}^{*}(\varphi)|\varphi, \tilde{\nu}\rangle
$$

The left and right eigenvectors form a complete basis

$$
\begin{aligned}
\sum_{\varphi, \nu}|\varphi, \nu\rangle\langle\varphi, \tilde{\nu}| & =1 \\
\left\langle\varphi, \tilde{\nu} \mid \varphi^{\prime}, \nu^{\prime}\right\rangle & =\delta_{\varphi, \varphi^{\prime}} \delta_{\nu, \nu^{\prime}}
\end{aligned}
$$

Turning back to the full lattice, disregarding normalization and gauge issues, the Bloch states can be written in the traditional way as a modulated plane wave:

$$
\langle n \mid k, \nu\rangle=\left\langle n \bmod (N) \mid \varphi_{k}, \nu\right\rangle \mathrm{e}^{i k n}
$$

where $\varphi_{k}$ is related to $k$ as defined previously. Consequently the time dependent solution of the rate equation is

$$
\left.p_{n}(t)=\sum_{k, \nu} \mathrm{e}^{-\lambda_{\nu}(k) t}\langle n \mid k, \nu\rangle\langle k, \tilde{\nu}| \text { initial-state }\right\rangle
$$

Averaging the probability within each unit cell, we get rid of the intra-cell modulation, leading to

$$
p_{n}(t) \approx \frac{1}{L} \sum_{k, \nu} C_{k, \nu} \mathrm{e}^{-\lambda_{\nu}(k) t} \mathrm{e}^{i k n}
$$

where $L$ is the length of the sample, and $C_{k, \nu}$ are constants that depend on the initial preparation. Note that in the limit $k \rightarrow 0$ the lower bands degenerate, reflecting a unique NESS. Furthermore, due to normalization $C_{0,0}=C=1$. The moment generating function that is associated with $p_{n}(t)$ is

$$
Z(k)=\sum_{n} \mathrm{e}^{-i k n} p_{n}(t) \approx \sum_{\nu} C_{k, \nu} \mathrm{e}^{-\lambda_{\nu}(k) t}
$$


The first and second moments of $n$ can be deduced by taking the first and second derivative of $Z(k)$ at $k=0$. In the long time limit, only the $\nu=0$ band survives. Expanding to second order in $k$ we get

$$
Z(k) \approx\left[C+C^{\prime} k+\frac{1}{2} C^{\prime \prime} k^{2}+\ldots\right]\left[1-\left(\lambda^{\prime} k+\frac{1}{2} \lambda^{\prime \prime} k^{2}+\ldots\right) t+\frac{1}{2}\left(\lambda^{\prime} k+\ldots\right)^{2} t^{2}+\ldots\right]
$$

From which we deduce that in the long time limit

$$
\begin{aligned}
\langle n\rangle & \approx i\left(C^{\prime}-C \lambda^{\prime} t\right) \\
\left\langle n^{2}\right\rangle & \approx-\left(C^{\prime \prime}-C \lambda^{\prime \prime} t-2 C^{\prime} \lambda^{\prime} t+C \lambda^{\prime 2} t^{2}\right) \\
\operatorname{Var}(n) & \approx C \lambda^{\prime \prime} t-C^{\prime \prime}+C^{\prime 2}
\end{aligned}
$$

where $C=1$, while $\lambda^{\prime}$ and $\lambda^{\prime \prime}$ are Taylor coefficients in the expansion of $\lambda_{0}(k)$. The mobility and the diffusion coefficient are determined accordingly:

$$
\begin{aligned}
v & =\lim _{t \rightarrow \infty}\left[\frac{\langle n\rangle}{t}\right]=\left.i \frac{\partial \lambda_{0}(k)}{\partial k}\right|_{k=0} \\
D & =\lim _{t \rightarrow \infty}\left[\frac{1}{2} \frac{\operatorname{Var}(n)}{t}\right]=\left.\frac{1}{2} \frac{\partial^{2} \lambda_{0}(k)}{\partial k^{2}}\right|_{k=0}
\end{aligned}
$$

\section{Appendix B: Calculation of $v$ and $D$ for $N=2$ lattice}

For the two site system, the lowest eigenvalue $\lambda_{0}(k)$ is

$$
\begin{aligned}
\lambda_{0}(k) & =\frac{1}{2}\left[(\vec{A}+\overleftarrow{A}+\vec{B}+\overleftarrow{B})-\sqrt{(\vec{A}+\overleftarrow{A}+\vec{B}+\overleftarrow{B})^{2}-4\left(1-\mathrm{e}^{-i \varphi}\right) \overleftarrow{A} \overleftarrow{B}-4\left(1-\mathrm{e}^{i \varphi}\right) \vec{A} \vec{B}}\right] \\
& \approx-i\left[\frac{\vec{A} \vec{B}-\overleftarrow{A} \overleftarrow{B}}{\vec{A}+\overleftarrow{A}+\vec{B}+\overleftarrow{B}}\right] \varphi+\left[\frac{\vec{A} \vec{B}+\overleftarrow{A} \overleftarrow{B}}{2(\vec{A}+\overleftarrow{A}+\vec{B}+\overleftarrow{B})}-\frac{(\vec{A} \vec{B}-\overleftarrow{A} \overleftarrow{B})^{2}}{(\vec{A}+\overleftarrow{A}+\vec{B}+\overleftarrow{B})^{3}}\right] \varphi^{2}
\end{aligned}
$$

From which the mobility and diffusion coefficients are derived

$$
\begin{aligned}
v & =\frac{\vec{A} \vec{B}-\overleftarrow{A} \overleftarrow{B}}{\vec{A}+\overleftarrow{A}+\vec{B}+\overleftarrow{B}} \\
D & =\frac{\vec{A} \vec{B}+\overleftarrow{A} \overleftarrow{B}}{2(\vec{A}+\overleftarrow{A}+\vec{B}+\overleftarrow{B})}-\frac{(\vec{A} \vec{B}-\overleftarrow{A} \overleftarrow{B})^{2}}{(\vec{A}+\overleftarrow{A}+\vec{B}+\overleftarrow{B})^{3}}
\end{aligned}
$$

